\title{
Satisfactory role of LEDs as a light receiving component and their uses in science demonstration experiments for educational purposes
}

\section{Makoto Hasegawa}

Makoto Hasegawa, "Satisfactory role of LEDs as a light receiving component and their uses in science demonstration experiments for educational purposes," Proc. SPIE 11143, Fifteenth Conference on Education and Training in Optics and Photonics: ETOP 2019, 111432F (2 July 2019); doi: $10.1117 / 12.2523733$ 


\title{
Satisfactory role of LEDs as a light receiving component and their uses in science demonstration experiments for educational purposes
}

\author{
Makoto Hasegawa* \\ Chitose Institute of Science and Technology, 758-65 Bibi, Chitose, Hokkaido, Japan 66-8655
}

\begin{abstract}
Light emitting diodes (LEDs) can be used also as a light-receiving element which is less likely to be saturated than photodiodes. The author has actually employed LEDs for the purpose of receiving optical signals in a science demonstration experiment set of optical communication for educational purposes in various ages. In some cases, LEDs as a light-receiving element can realize better quality of reproduced sounds transmitted in the demonstration experiment set via optical transmission, as compared to the case where a normal photodiode is employed as a light-receiving element. Furthermore, by using LEDs both for a light source and a light-receiving component, relationships among color of light (wavelengths or frequencies) and their energies as well as bandgap energies of semiconductor materials can be explained in such demonstration. In this paper, some exemplary data of output voltage characteristics obtained from an LED employed as a light-receiving element, as well as typical demonstration sets for educational purposes, are explained.
\end{abstract}

Keywords: LED, optical communication, light receiving component, demonstration experiment, physics education

\section{INTRODUCTION}

Light emitting diodes (LEDs) are widely being used in our modern life as light sources for illumination. Since LEDs have already become popular even for children, they are often used in science demonstration experiments and classroom experiments as handy light sources. On the other hand, LEDs can also act as light-receiving elements or light detectors. This is because LEDs have a p-n junction. Normally, the p-n junction in LEDs acts as key mechanism for emitting light beams once a current is allowed to flow into there. At the same time, just similar to photodiodes (PD), once light beams are allowed to reach the p-n junction in LEDs, a certain amount of current/voltage is generated there and can be obtained as an output signal. Several papers reported exemplary light-receiving applications of LEDs ${ }^{1-10}$.

The author paid attention to such light-receiving performances of LEDs, and employed them in a science demonstration experiment set of optical communication for educational purposes, both as a light source and as a light-receiving element ${ }^{11-15}$. Under certain situations, an LED acting as a light-receiving element can realize clearer reproduction of transmitted audio signals (music), as compared to the case where a normal PD is employed as a light-receiving element, in such an optical communication demo set. Furthermore, by using LEDs both for a light source and a light-receiving component, relationships among color of light (wavelengths or frequencies) and their energies as well as bandgap energies of semiconductor materials can be explained in such demonstration.

In this paper, some exemplary data of output voltage characteristics obtained from an LED employed as a light-receiving element are shown and compared to the PD performances. Typical demonstration sets for optical communication to be used for educational purposes are also explained.

\section{EXPERIMENTAL DATA OF LEDS AS A LIGHT-RECEIVING ELEMENT}

In order to demonstrate light-receiving capabilities of LEDs, some experimental data are shown below. Two types of commercially-available red-color LEDs were employed.

-Type A: wavelength 630nm, viewing angle 8 degrees, intensity 6000mcd@20mA, $\mathrm{V}_{\mathrm{F}}=1.9 \mathrm{~V}$

-Type B: wavelength 630nm, viewing angle 20 degrees, intensity $4000 \mathrm{mcd} @ 20 \mathrm{~mA}, \mathrm{~V}_{\mathrm{F}}=1.9 \mathrm{~V}$

*hasegawa@phton.chitose.ac.jp; phone/fax +81 123276059

Fifteenth Conference on Education and Training in Optics and Photonics: ETOP 2019, edited by

Anne-Sophie Poulin-Girard, Joseph A. Shaw, Proc. of SPIE Vol. 11143, 111432F · (c) 2019

SPIE, ICO, IEEE, OSA · CCC code: 0277-786X/19/\$18 · doi: 10.1117/12.2523733 
Both of the employed LEDs have almost the same operating specifications, except that Type 2 LED has a slightly wider viewing angle than Type 1 LED.

One LED of each of the above two types was incorporated into a typical LED operating circuit with a power supply of DC5V. The operating circuit also included a $100 \Omega$ resistor, a variable $500 \Omega$ resistor, and a variable $10 \mathrm{k} \Omega$ resistor, connected in series with the LED. Among these three resistors, the two variable resistors were used for adjusting an operating current level to change an intensity of light to be emitted from the LED, while the $100 \Omega$ resistor was employed for limiting the current level. A current meter was also connected in the operating circuit to measure the operating current levels.

In order to confirm whether the light intensity emitted from each type of those LEDs was actually in proportion to the operating current level, the emitted light beams were first allowed to be incident onto a light power meter, and its output levels were measured while changing the operating current levels of the light source LED. The distance L between the light source LED and the light power meter was set at $2 \mathrm{~cm}, 5 \mathrm{~cm}, 10 \mathrm{~cm}$, and $20 \mathrm{~cm}$. The obtained results are shown in Fig.1. Both of the two types of LEDs showed satisfactory linear characteristics.

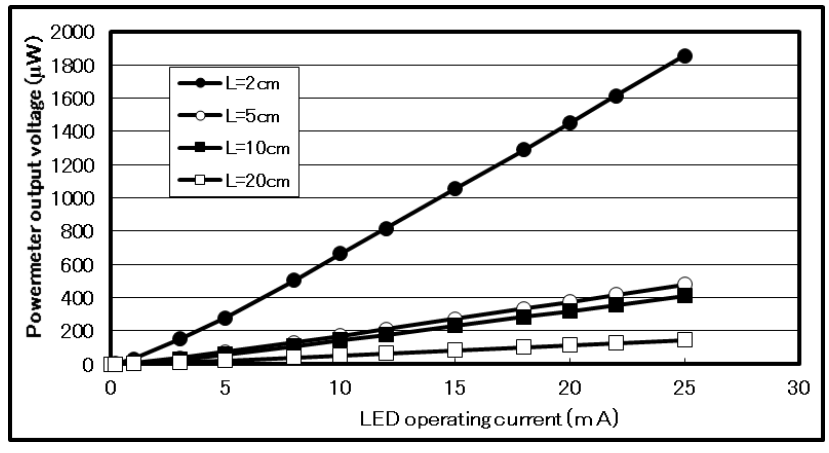

(a) Type A LED

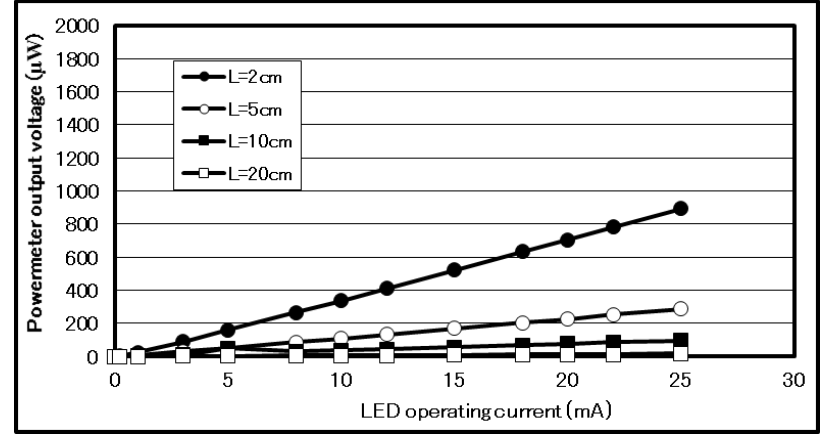

(b) Type B LED

Figure 1. Relationship between the operating current levels of the light source LED and the output levels from the light powermeter irradiated with the emitted light beams.

The power meter was then replaced with a PD, and the output characteristics of the respective types of LEDs were similarly measured. It should be noted that it is intended to realize an optical communication demonstration set for receiving audio signals (music) which will be then reproduced from a loud speaker. Therefore, for the measurements, the two terminals of the PD were directly connected to a monaural mini plug jack which was in turn to be connected to a loudspeaker. An output voltage signal from the monaural mini plug jack without the loudspeaker connected was measured with a storage scope. The results are shown in Fig.2. Certain saturation tendencies can be recognized for both LED types.

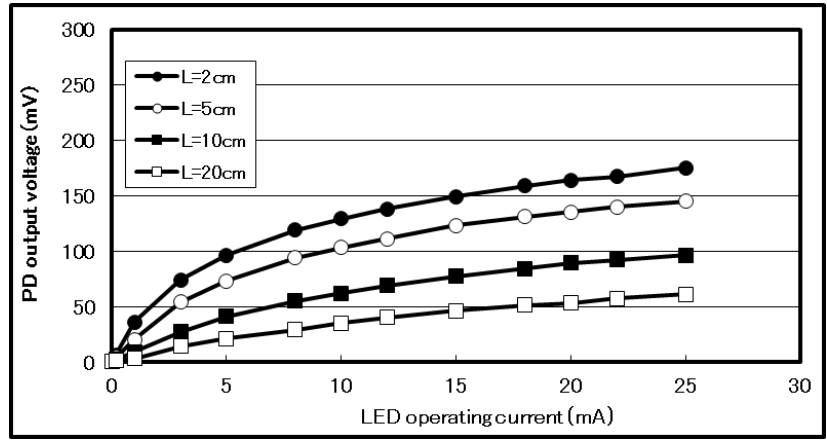

(a) Type A LED

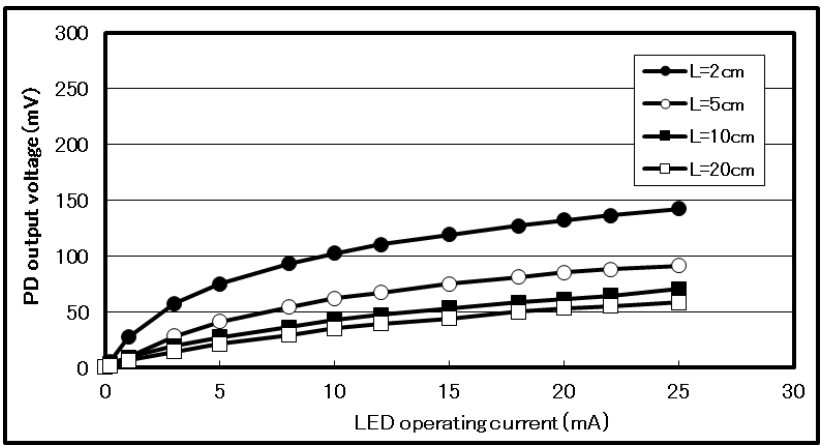

(b) Type B LED

Figure 2. Relationship between the operating current levels of the light source LED and the output levels from the photodiode (PD) irradiated with the emitted light beams. 
Then, the PD was replaced with another piece of the same LED type as employed as the light source, respectively, and the similar measurements were further conducted. The obtained results are shown in Fig.3. Capabilities of the LEDs as a light-receiving element can be clearly recognized.

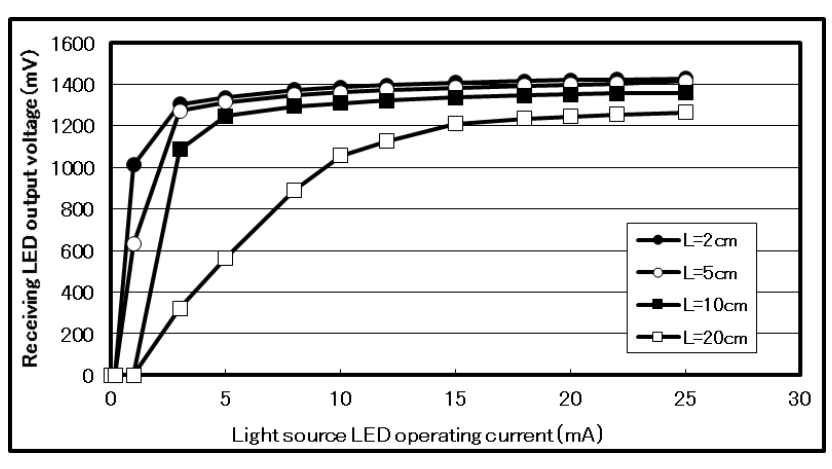

(a) Type A LED

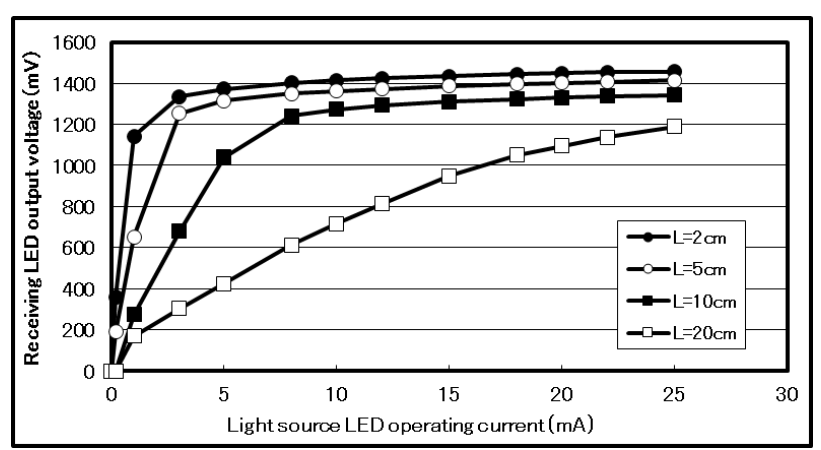

(b) Type B LED

Figure 3. Relationship between the operating current levels of the light source LED and the output levels from the LED as a lightreceiving element irradiated with the emitted light beams.

As previously described, data in Figs.2-3 were obtained without any loudspeaker connected to the monaural mini plug jack to which the PD/receiving LED were connected. In order to obtain data in more actual situation to be encountered in typical actual optical communication demonstrations, the similar measurements were further conducted while a loudspeaker (with an input impedance of $10 \mathrm{k} \Omega$ ) was connected to the monaural mini plug jack (but not in operation) with the distance $\mathrm{D}$ up to $10 \mathrm{~cm}$.

The obtained results are shown in Fig.4 and Fig.5, respectively.

In this cases, when a PD was used as the light receiving element (see Fig.4), saturation tendencies in the output voltages became significant with the smaller distance D between the light source LED and the PD. In contrast, when an LED was used also as the light-receiving element (see Fig.5), no such saturations in the output voltages were found even when the light-receiving LED was placed relatively close to the light source LED.

When considering actual application in an optical communication demonstration set, availability of the output signals without any saturations is very advantageous, because clear reproductions of audio signal (such as music) become possible. Therefore, the performance characteristics as shown in Fig.5 can be significant benefits for employing an LED also as a light-receiving element in optical communication demonstration.

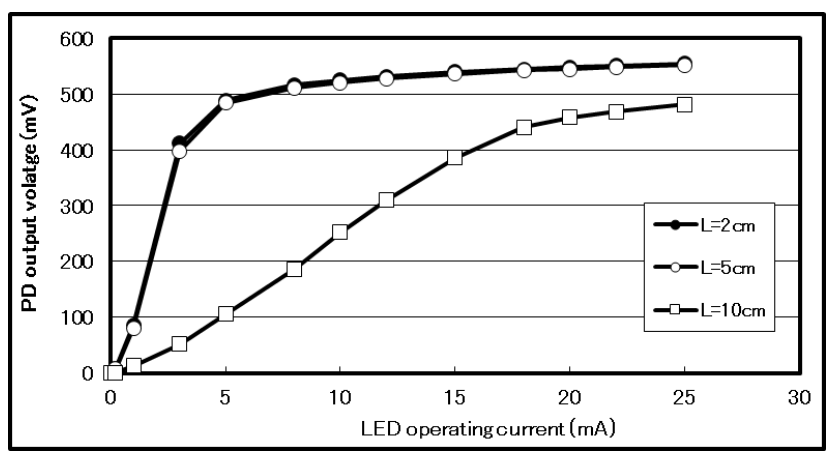

(a) Type A LED

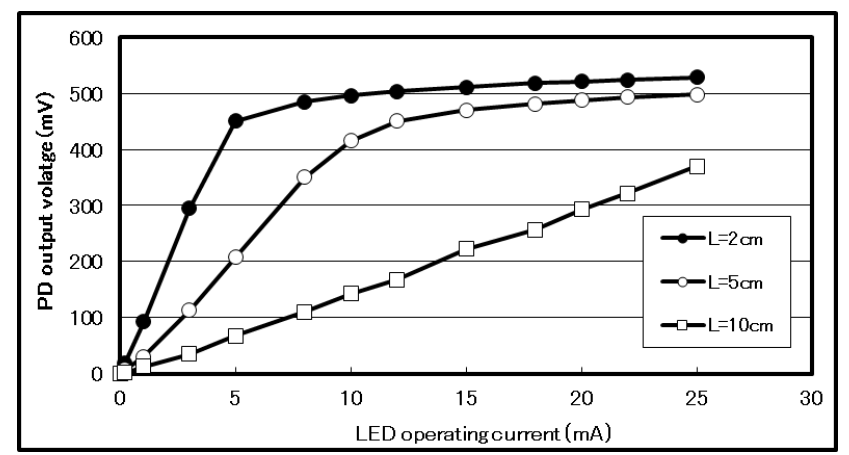

(b) Type B LED

Figure 4. Relationship between the operating current levels of the light source LED and the output levels from the photodiode (PD) irradiated with the emitted light beams with a loudspeaker connected. 


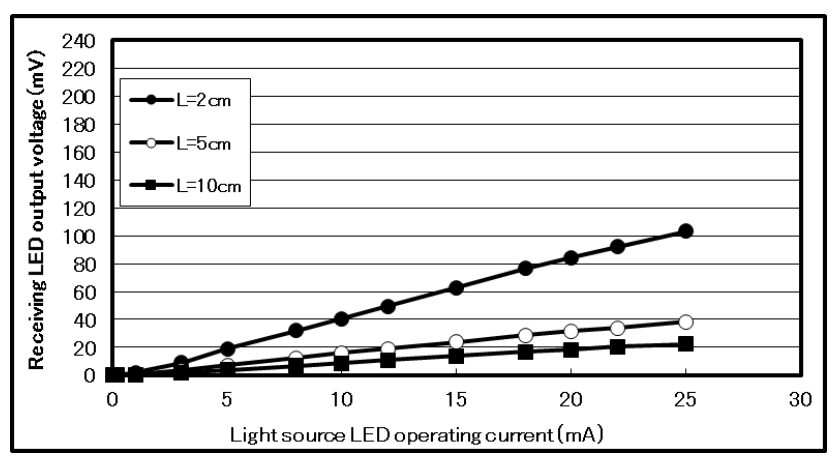

(a) Type A LED

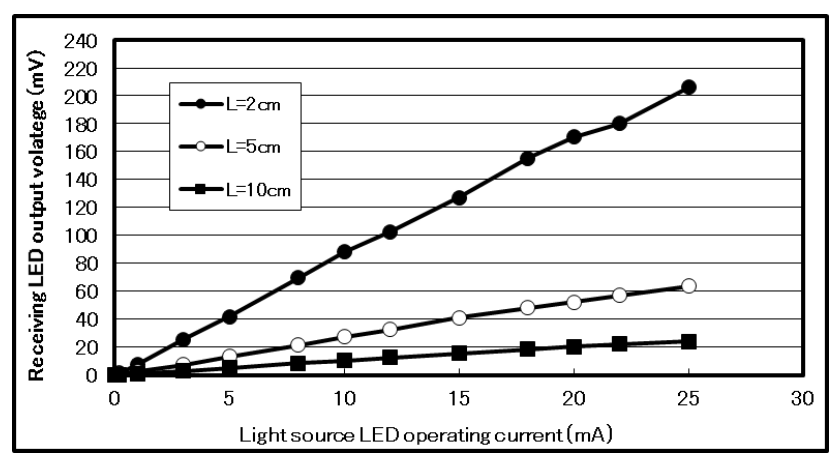

(b) Type B LED

Figure 5. Relationship between the operating current levels of the light source LED and the output levels from the LED as a lightreceiving element irradiated with the emitted light beams with a loudspeaker connected.

\section{OPTICAL COMMUNICATION DEMONSTRATION SET WITH LEDS AS BOTH A LIGHT SOURCE AND A LIGHT-RECEIVING ELEMENT}

In this section, a typical optical communication demonstration set with LEDs both as a light source (light-transmitting element) and a light-receiving element is explained. This demonstration set is basically the same as those described in the author's previous reports ${ }^{11-14}$ and is intended to be employed in science experimental demonstration events and/or science classes for educational purposes.

Fig.6 shows a basic circuit diagram for a light transmitting section (left) and a light receiving section (right) of an experiment set for optical communication demonstration. Fig.7 show a photo of exemplary arcual circuits (left: a light transmitting circuit, right: a light receiving circuit).

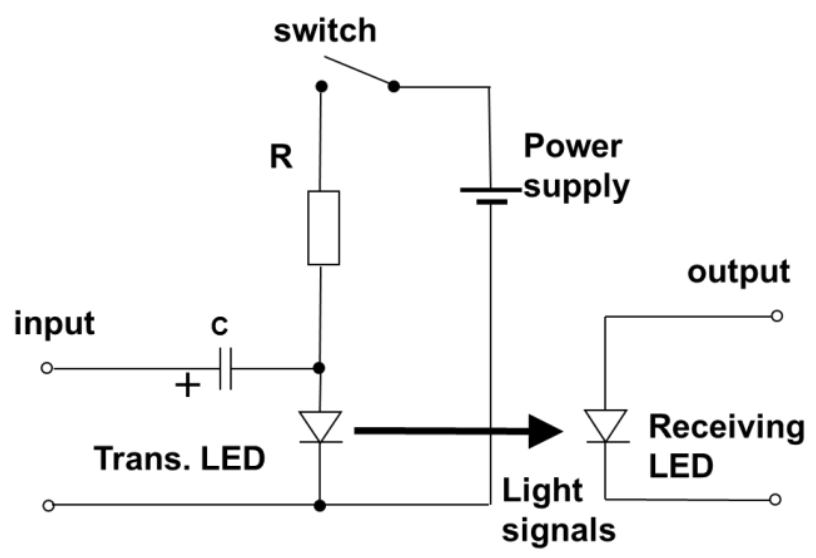

Figure 6. Circuit diagrams of an experimental set for optical communication demonstration.

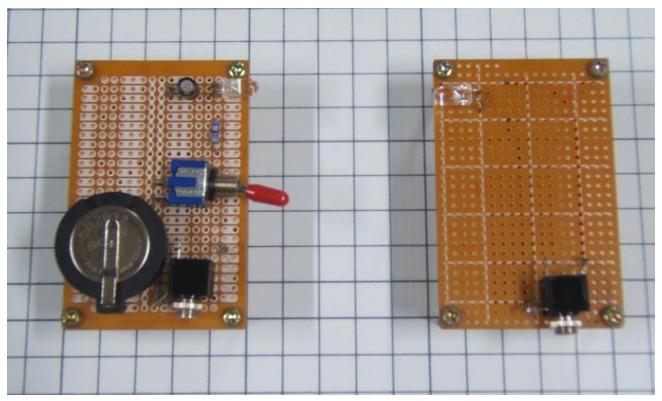

Figure 7. A photo of exemplary arcual circuits (left: a light transmitting circuit, right: a light receiving circuit). 
In a light transmitting circuit, a usual battery is employed for supplying power to an LED. While the circuit as shown in Fig.7 includes one $3 \mathrm{~V}$ battery, combination of two $1.5 \mathrm{~V}$ batteries is also applicable. A resistor is connected for limiting a current flowing through the LED and its resistance value can be determined by well-known calculation. For example, when an LED with a forward voltage $\mathrm{V}_{\mathrm{F}}=1.9 \mathrm{~V}$ and a nominal operating current $\mathrm{I}_{\mathrm{p}}=20 \mathrm{~mA}$ is connected with a battery $\mathrm{V}_{\mathrm{S}}=3 \mathrm{~V}$, a resistance value $\mathrm{R}$ can be determined as $\mathrm{R}=\left(\mathrm{V}_{\mathrm{S}}-\mathrm{V}_{\mathrm{F}}\right) / \mathrm{I}_{\mathrm{P}}=$ about $50 \Omega$. When audio input signals are not supplied, the LED in this circuit configuration will be operated with a DC current from the DC power supply (batteries) in a usual manner.

For realizing optical communication demonstration, audio signals are provided to an input terminal. Typically, music signals are provided from a CD player. This input terminal can be a monaural mini plug jack to be connected with an audio signal source such as an earphone terminal of the $\mathrm{CD}$ player. The audio input signals are superimposed over a DC operating current supplied from the battery to the LED, so that amplitudes of the DC operating current for the LED can be modulated in accordance with the supplied audio input signals. In other words, amplitude modulation of the LED light beams with the audio signals is realized. A capacitor (particularly, $22 \mu \mathrm{F}$ in this case) is intended to cut DC noises in the audio signal.

The modulated light beams emitted from the LED in the light transmitting circuit is then received by a light-receiving LED in the light receiving circuit. Alternatively, a normal PD can be of course used as the light receiving element. The detected light signals are converted into electric signals and provided to a loudspeaker for audio reproduction. No power supply is provided in the light receiving circuit, and the light-receiving element (LED or PD) is directly connected to a monaural mini plug jack as an output terminal. By employing a loudspeaker incorporating an amplifier, no amplification function is necessary in the circuit, and the output signal from the light-receiving element can be directly provided to the loudspeaker.

\section{ACTUAL DEMONSTRATIONS}

The optical communication demonstration set has been often used in various situations by the author myself as well as by the members of the student project team "Rika-Kobo" of the author's university ${ }^{14-18}$.

One of the typical situations is a science experiment demonstration event. Typically, a light transmitting circuit and a light receiving circuit are positioned on a table with a distance in the rage of about 5 to $15 \mathrm{~cm}$ from each other. Amplitudes of the LED light beams are modulated with music signals from a CD player and transmitted, and then received by the light-receiving LED in the counterpart light receiving circuit. As a result, music can be reproduced from a loudspeaker. Interrupting light transmission between the two LEDs with hand or any other appropriate material causes music reproduction to be stopped, which gives good surprises to audience in various generations from children to their parents and/or seniors. Due to non-saturated performances as mentioned previously, clearer music reproduction can be often realized by employing, instead of a PD, the LED as a light-receiving element.

Another typical situation for optical communication demonstration is a science class targeted from elementary school children to junior-high school or high school students. For example, in classes for elementary school children, after demonstration showing the operations of the demonstration set (Fig.8(a)), children themselves can make up their own circuit kit as a group work and confirm the operation (Fig.8(b)).

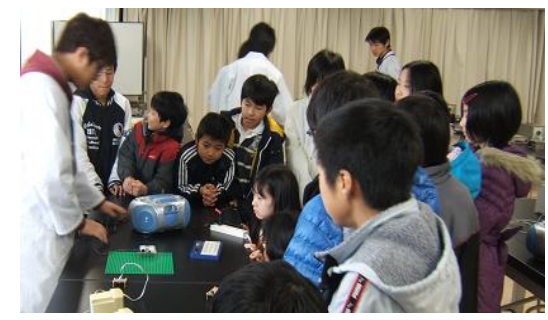

(a) Explanation of operations

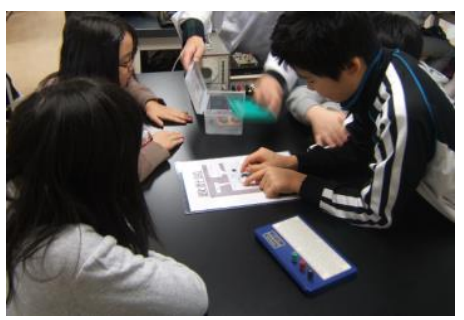

(b) group work for making up the circuit

Figure 8. Science class for elementary school children using the optical communication demondtrstion set.

For high school students or other people who have some or certain knowledges on LEDs and know their function as light emitting components, unexpected effects of light receiving performances of LEDs can be effective to let them get interested and encourage them to obtain further knowledges and explanations on the functions. 
When the students have knowledge about relationships between colors of light (wavelengths or frequencies) and bandgap energies of semiconductor materials, then further advanced explanations can be made through appropriate selections of LEDs with different colors as the light transmitting and receiving elements. Specifically, appropriate combinations of colors can selectively realize successful transmission and reproduction of audio signals (music). For example, when a blue-color LED is selected as a light source and a red-color LED is selected as a light-receiving element, successful music reproduction is realized. However, reverse selection leads to unsuccessful result. Such demonstration can act as a useful introductory tool for explaining relationships among colors of light (wavelengths or frequencies) from LEDs, their energy levels and band gap energies of semiconductor materials used in the LEDs.

Furthermore, as a modified version ${ }^{14}$, simplified demonstration for wavelength multiplied optical communication can also be prepared with a white-color LED, for example, as the one shown in Fig.9. In this case, a white-color LED including a red-color LED element, a green-color LED element and a blue-color LED element is employed as a light source, and operating current levels for these LED elements are independently modified in accordance with different audio signals. When receiving the modulated light, three different audio signals are simultaneously demodulated. Alternatively, by employing appropriate filters, one among these three audio signals can be selected to be reproduced. Such a demonstration set is also effective and useful for explaining primary colors of light. It should be noted, however, that in this case, use of a PD or a solar cell panel as a light receiving element is necessary.

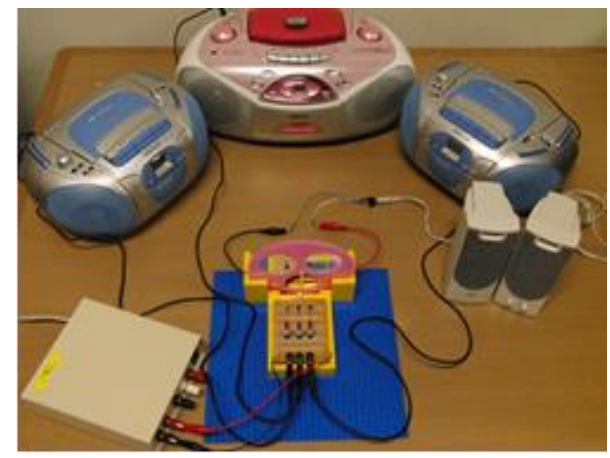

Figure 9. A simplified demonstration set for wavelength multiplied optical communication with a white-color LED.

\section{SUMMARY}

LEDs can be also employed as a light-receiving element, and such use can exibit several advantages compared to the conventional photodiode. A simple experiment set for demonstrating optical communication can be provided in which LEDs are employed both as a light source and as a light-receiving element. This experiment set has been effectively used in various situations of science demonstrations, including science classes for elementary school children to high school students, as well as other educational facilities. Various levels from simple demonstration of optical communication up to explanations of relationships between colors of light (wavelengths or frequencies) and bandgap energies of semiconductor materials can be successfully conducted.

\section{REFERENCES}

[1] Mims III, F.M., "Sun photometer with light-emitting diodes as spectrally selective detectors," Appl. Opt., Vol.31, No.33, pp.6965-6967 (1992).

[2] Mims III, F.M., "Solar radiometer with light-emitting diodes as spectrally-selective detectors," Engineering \& Laboratory Notes, Supplement to Optics \& Photonics News, Vol.11, No.2, pp.3-4 (2000).

[3] Miyazaki, E., Itami, S. and Araki, T., "Using a light-emitting diode as a high-speed, wavelength selective photodetector," Review of Scientific Instruments, Vol.69, No.11, pp.3751-3754.

[4] Brooks, D.R. and Mims III, F.M., "Development of an inexpensive handheld LED-based Sun photometer for the GLOBE program," J. Geophys. Res., Vol.106, No.D5, pp.4733-4740 (2001).

[5] Mims III, F.M., "An inexpensive and stable LED Sun photometer for measuring the water vapor column over south Texas from 1990 to 2001," Geophys. Res. Lett., Vol.29, No.13, p.1642 (2002). 
[6] Acharya, Y.B., "Spectral and emission characteristics of LED and its application to LED-based sunphotometry," Optics \& Laser Technology, Vol.37, pp.547-550 (2005).

[7] Czapla-Myers, J.S., Thome, K.J. and Biggar, S.F., "Design, calibration, and characterization of a field radiometer using light-emitting diodes as detectors," Appl. Opt., Vol.47, No.36, pp.6753-6762 (2008).

[8] Ben-Ezra, M., Wang, J., Wilburn, B., Li, X. and Ma, L., "An LED-only BRDF measurement device," 2008 IEEE Conf. on Computer Vision \& Pattern Recognition (2008), DOI:10.1109/CVPR.2008.4587766.

[9] Mims III, F.M., "LED Sun photometry," Optics \& Photonics News, Vol.20, No.9, pp.32-38 (2009).

[10] Kowalczyk, M. and Siuzdak, J., "Photo-reception properties of common LEDs," Opto-Electronics Review, Vol.25, pp.222-228 (2017).

[11] Hasegawa, M., "A simplified experiment set for demonstrating optical communication with LEDs as light transmitting and receiving components," Proc. of 2013 Intl. Conf. on Physics Education (ICPE-EPEC2013), no.093.P1, pp.1151-1157 (2013).

[12] Hasegawa, M., "Use of LEDs as a light receiving component and their application to demonstration experiments for educational purposes," Proc. of 2015 IEEE Intl. Conf. on Teaching, Assessment, and Learning for Engineering (TALE2015), no.113, pp.31-35 (2015).

[13] Hasegawa, M., "Application of optical communication demonstration set employing LEDs both as light transmitting and receiving elements for education in various generations," Trans. Mat. Res. Soc. Japan (Transactions of the Materials Research Society of Japan), Vol.41, No.1, pp.17-20 (2016).

[14] Hasegawa, M. and Tokumitsu, S., "Continuous outreach activities performed by a student project team of undergraduates and their program topics in optics and photonics", Proc. of SPIE vol.9946, pp.99460D-1 99460D-9, (SPIE Optics+Photonics, Optical Engineering+Applications, Optics Education \& Outreach IV, paper no.9946-12), (2016).

[15] Hasegawa, M., "Roles and effects of activities of a student project team in engineering education for university students in lower grades," Proc. of 2013 IEEE Intl. Conf. on Teaching, Assessment, and Learning for Engineering (TALE2013), no.140, pp.87-90 (2013).

[16] Hasegawa, M., "New education scheme for college students through out-of-curriculum project activities," Intl. Journal of Modern Education Forum (IJMEF), Vol.4, Issue 3, pp.120-123 (2014).

[17] Hasegawa, M., "Case study on educational effects for university students of their out-of-curriculum project activities," Proc. of 2015 2nd Intl. Conf. on Educational Reform and Modern Management (ERMM2015), no.ERMM2015-E040, pp.205-208 (2015).

[18] Hasegawa, M., "Engineering educational effects for undergraduate students through out-of-curriculum project activities," Proc. of Intl. Conf. on Electrical Engineering (ICEE2016), no.90064 (2016). 